\title{
Two-Stage Latissimus Dorsi Flap with Implant for Unilateral Breast Reconstruction: Getting the Size Right
}

\author{
Jiajun Feng ${ }^{1}$, Cleone I Pardoe ${ }^{2}$, Ashley Manuel Mota ${ }^{3}$, Christopher Hoe Kong Chui ${ }^{1}$, \\ Bien-Keem Tan ${ }^{1}$ \\ ${ }^{1}$ Department of Plastic, Reconstructive and Aesthetic Surgery, Singapore General Hospital, Singapore; ${ }^{2}$ College of Medical and Dental \\ Sciences, University of Birmingham, Birmingham; ${ }^{3} \mathrm{Hull}$ and East Yorkshire Trust, England, Yorkshire, UK
}

\begin{abstract}
Background The aim of unilateral breast reconstruction after mastectomy is to craft a naturallooking breast with symmetry. The latissimus dorsi (LD) flap with implant is an established technique for this purpose. However, it is challenging to obtain adequate volume and satisfactory aesthetic results using a one-stage operation when considering factors such as muscle atrophy, wound dehiscence and excessive scarring. The two-stage reconstruction addresses these difficulties by using a tissue expander to gradually enlarge the skin pocket which eventually holds an appropriately sized implant.

Methods We analyzed nine patients who underwent unilateral two-stage LD reconstruction. In the first stage, an expander was placed along with the LD flap to reconstruct the mastectomy defect, followed by gradual tissue expansion to achieve overexpansion of the skin pocket. The final implant volume was determined by measuring the residual expander volume after aspirating the excess saline. Finally, the expander was replaced with the chosen implant.

Results The average volume of tissue expansion was $460 \mathrm{~mL}$. The resultant expansion allowed an implant ranging in volume from 255 to $420 \mathrm{~mL}$ to be placed alongside the LD muscle. Seven patients scored less than six on the relative breast retraction assessment formula for breast symmetry, indicating excellent breast symmetry. The remaining two patients scored between six and eight, indicating good symmetry.

Conclusions This approach allows the size of the eventual implant to be estimated after the skin pocket has healed completely and the LD muscle has undergone natural atrophy. Optimal reconstruction results were achieved using this approach.
\end{abstract}

Keywords Breast / Reconstructive surgical procedures / Tissue expansion / Surgical flaps / Radiotherapy
Correspondence: Bien-Keem Tan Department of Plastic, Reconstructive and Aesthetic Surgery, Singapore General Hospital, Outram Road, Singapore 169608, Singapore

Tel: $+65-6321-4686$

Fax: +65-6227-3573

E-mail: bienkeem@gmail.com
We thank Mr. Evan Lim for the medical illustration.

No potential conflict of interest relevant to this article was reported.

Received: 29 Jul $2015 \bullet$ Revised: 3 Oct $2015 \bullet$ Accepted: 5 Nov 2015

pISSN: 2234-6163 • elSSN: 2234-6171 • http://dx.doi.org/10.5999/aps.2016.43.2.197 • Arch Plast Surg 2016;43:197-203

\section{INTRODUCTION}

One of the most challenging goals in unilateral breast reconstruction using a latissimus dorsi (LD) flap with an implant is deter- mining and achieving the ideal breast volume. The components that contribute to breast volume are the LD muscle, its skin paddle, and the implant. Given the variability of autologous tissue volume and the likelihood of postoperative muscle atrophy, it is 
imperative to maximize the size of the implant in order to compensate for the inevitable volume loss within the first postoperative year.

Surgically, two options are available when using a LD flap with an implant: one-stage or two-stage reconstruction. With the use of a one-stage reconstruction the final volume is often insufficient [1]. We have observed that when a flap and implant combination is used, the thinness and tightness of the skin envelope after the mastectomy imposes a size limit on the implant. The mastectomy skin envelope must accommodate both the LD flap and the implant, and a large implant can exert excessive pressure on both the LD muscle and mastectomy skin envelope, causing ischemia and ultimately wound dehiscence. In order to maximize the size of the implant in a single-stage reconstruction, the breast envelope often needs to be enlarged with an LD skin paddle. However, this leads to unsightly scars.

The two-stage LD flap reconstruction can be used to circumvent these difficulties [2]. The first stage comprises the insertion of a LD flap and a tissue expander, followed by gradual skin expansion and replacement with the implant in the second stage. This paper presents our experience of using this technique, as exemplified by the case studies described.

\section{METHODS}

All patients signed a written consent form to allow their photographs and data to be used for research and education purposes. The data and photographs were de-identified.

All patients who received two-stage LD reconstruction after a mastectomy from September 2011 to December 2014 in Singapore General Hospital were included in this study. Prior to surgery, all patients underwent breast imaging, biopsy, and a metastatic work-up. Sentinel lymph biopsy or axillary clearance was performed in patients with a pre-surgical diagnosis of breast cancer. The mastectomy was followed by an LD flap with an expander reconstruction in the same operation. The need for adjuvant chemotherapy or radiotherapy was determined postoperatively according to the tumor staging.

The indication for this procedure was unilateral breast reconstruction in patients with medium to large breasts. These patients either wanted to avoid an abdominal donor site or had undergone previous abdominal operations. The goal was to match the volume and ptosis on the opposite side.

The relative breast assessment score (pBRA) was used to assess postoperative breast symmetry [3] using the patients' most recent frontal photographs. The score was calculated using the formula

$$
\mathrm{pBRA}=\left(\frac{\sqrt{(a 1-b 1)^{2}+(a 2-b 2)^{2}}}{\sqrt{\left(b 1^{2}+b 2^{2}\right)}}\right) \times 100,
$$

where $a 1$ is the horizontal distance between the nipple of the reconstructed breast and the midline; $b 1$ is the same on the contralateral breast; $a 2$ is the nipple level of the reconstructed breast, which is derived by measuring from the origin of a perpendicular line dropped from a horizontal cut through the sternal notch; and $b 2$ is the same on the opposite side. A score of six and below indicates excellent symmetry, while a score between six and eight indicates good symmetry and a score above eight indicates fair to poor symmetry.

\section{Surgical technique}

The first step of the two-stage reconstruction is to harvest the LD flap with a small skin paddle measuring $4-5 \mathrm{~cm}$ in width. A small paddle allows closure of the donor site with a short scar. The role of the skin paddle is to cover the nipple-areolar complex. Additional skin is de-epithelialized and buried, and the thoracodorsal nerve is spared to reduce muscle atrophy. A tissue

\section{Fig. 1. Two-stage LD reconstruction with an expander}

Inferior placement of the port through the inframammary fold. A skin paddle is used to cover the nipple-areolar complex defect. Anchoring sutures are placed along the borders of the latissimus dorsi (LD) muscle.

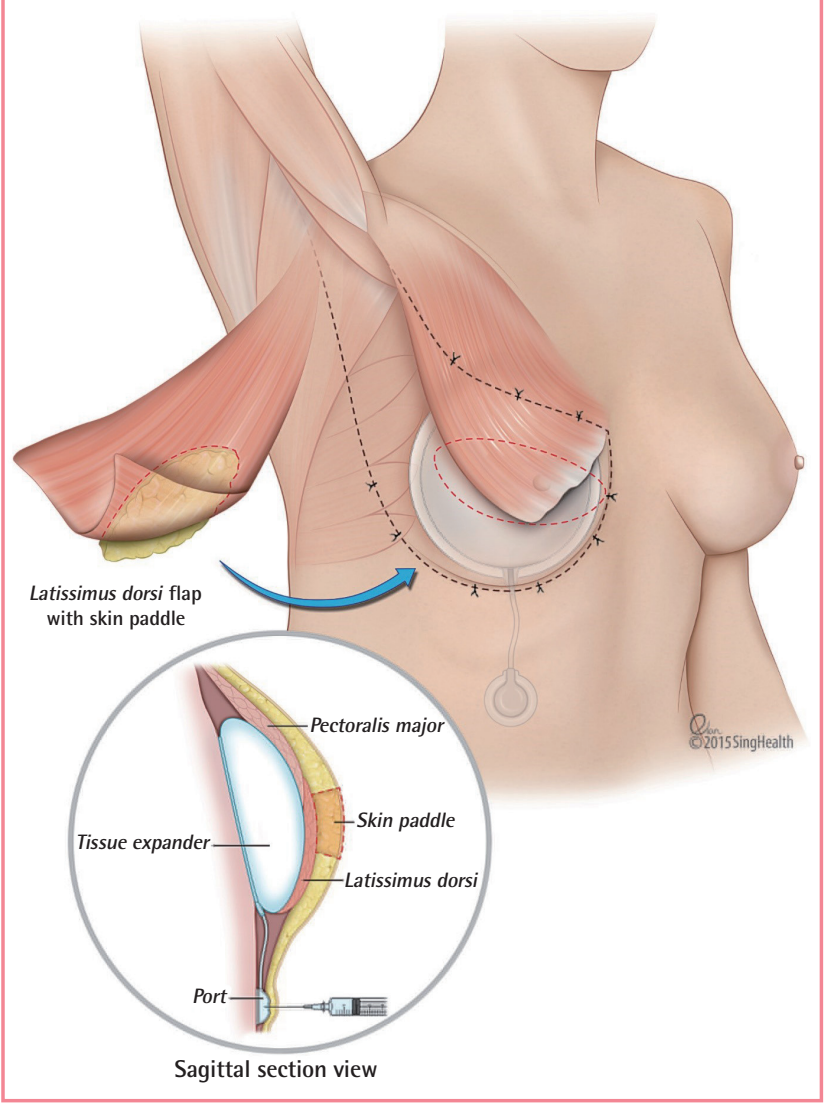


expander with a volume of 400 or $550 \mathrm{~mL}$ (Mentor, Irvine, California, USA) is inserted in the subpectoral plane. The injection port for the expander is placed in a subcutaneous tunnel that crosses the inframammary fold and is positioned overlying the costal cartilage (Fig. 1). Tunnel dissection is performed with Dilson Luz dilators to minimize damage to the inframammary fold. Avoiding a laterally placed injection port prevents communication between the breast skin pocket and the LD muscle donor site and also reduces the risk of port migration. The expander is pre-filled with a small volume $(20-200 \mathrm{~mL})$ of saline prior to placement to ensure tension-free closure of the breast skin pocket. The LD muscle is then used to cover the inferior aspect of the tissue expander. Anchoring sutures are placed at the borders of the LD muscle: superiorly to join the pectoralis major, medially to create the middle cleavage, laterally to prevent lateral migration of the expander, and inferiorly to define the inframammary fold (Fig. 1). Tissue expansion is initiated upon wound healing, usually at two to three weeks.

During the second stage, the patient receives weekly saline injections into the expander to gradually enlarge the skin pocket.

\section{Fig. 2. Two-stage LD reconstruction for patient C}

(A) Preoperative photograph. (B) Over-expansion with a tissue expander volume of $540 \mathrm{~mL}$. (C) The tissue expander deflated to $250 \mathrm{~mL}$ to achieve symmetrization. (D) Postoperative photograph at nine months after placement of a 255-mL Mentor implant and nipple reconstruction. LD, latissimus dorsi.
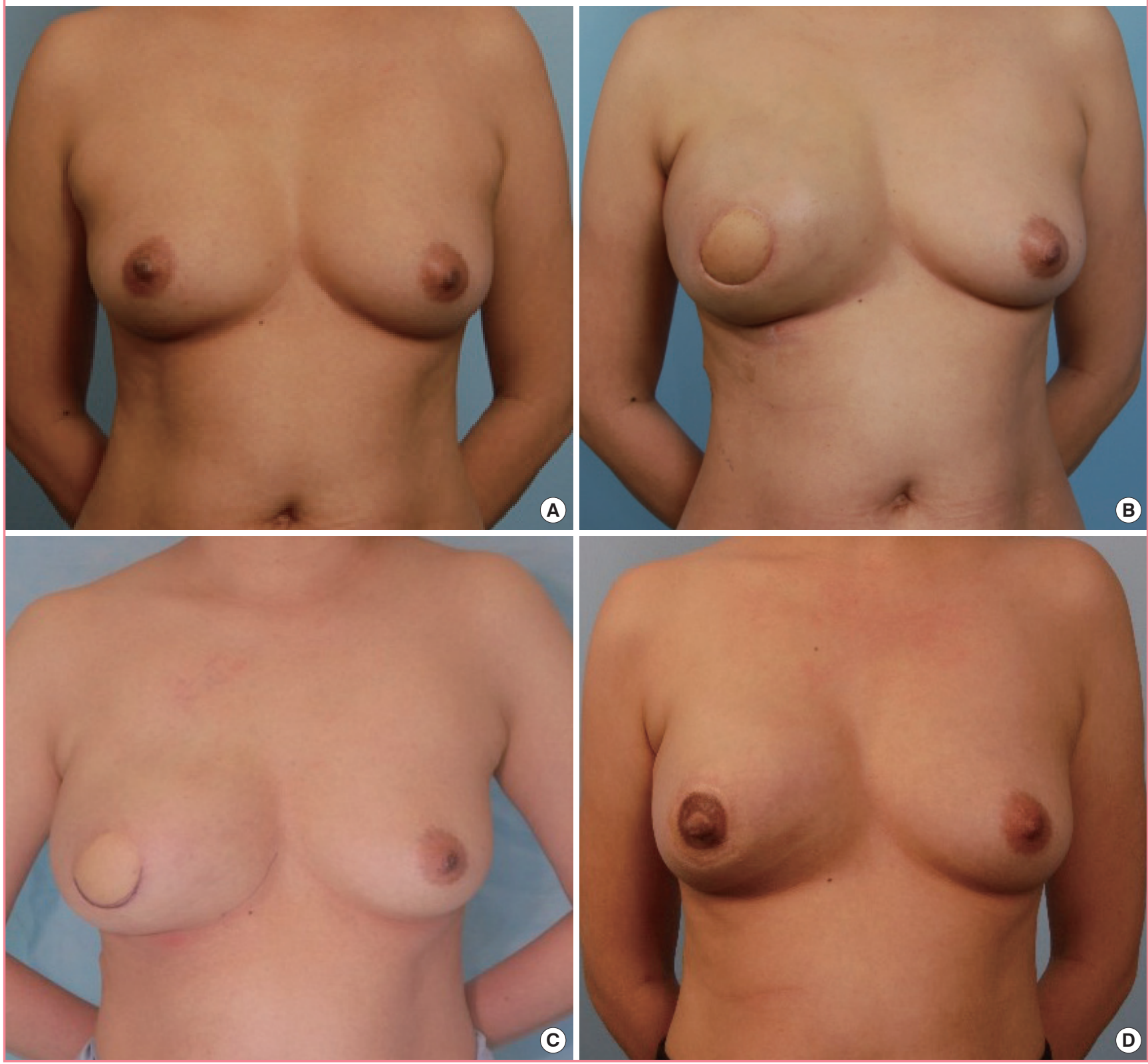
Aseptic technique is used to limit the risk of infection of the expander, which is paramount in patients undergoing chemotherapy because the immune system of such patients is already suppressed. Approximately $40-60 \mathrm{~mL}$ of saline is used for injection, an amount that is limited by the slight tightness of the skin pocket. Three to four months are necessary to reach the desired $20 \%-$ $30 \%$ level of overexpansion compared to the contralateral breast (Fig. 2B). As part of the preoperative workup for placement of the implant, the implant size needs to be determined. The size of the implant is estimated from the residual expander volume after the excess saline has been aspirated, achieving symmetry with the contralateral breast (Fig. 2C). The patient can watch the immediate result of the expander deflation and decide when

\section{Fig. 3. Breast prostheses volumes}

Volume of the tissue expander and implant size during two-stage reconstruction for the nine patients $(A-I)$.

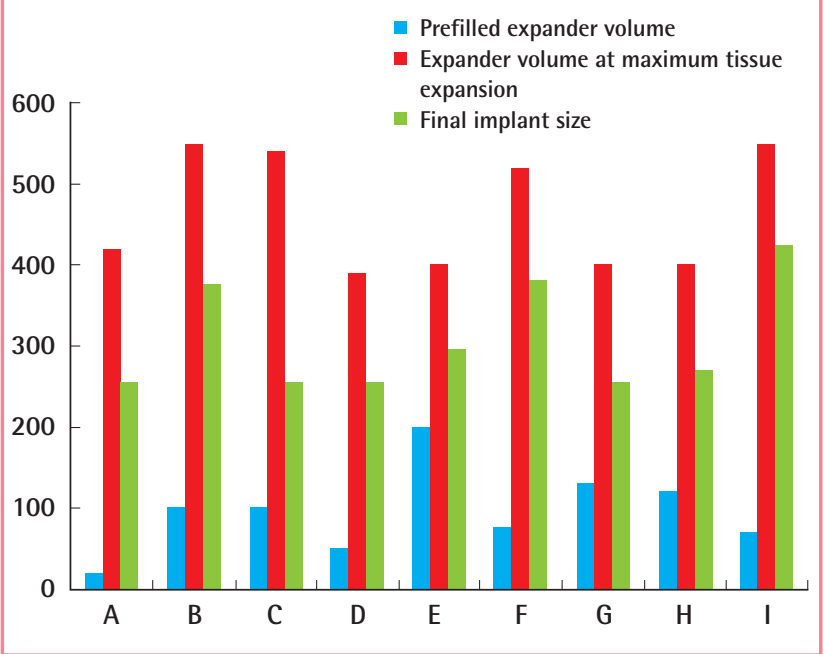

the size and symmetry are acceptable to her. The saline is then re-injected to maintain the overexpansion until the operation date. During the operation, the expander and adjoining port are removed, a pre-chosen implant (Allergan, Mentor, or Silimed) is placed, and the inframammary fold is repaired. If necessary, the skin pocket can be adjusted and nipple reconstruction performed in the same operation.

It should be noted that in patients treated with adjuvant radiotherapy, an initial partial expansion is performed, followed by full expansion upon completion of radiotherapy.

For patients undergoing adjuvant chemotherapy, the tissue expansion process can be performed, provided a suitable level of immunity exists; however, the implant placement must be delayed until chemotherapy is completed.

\section{RESULTS}

Nine patients were included in this study, with an average age of 48 years (range, 28-64 years). Eight patients had been diagnosed with primary breast cancer and the remaining case was a nonmalignant phyllodes tumor. All breast cancer patients were free of metastasis at the time of surgery and had received a skin-sparing mastectomy with curative intent. Three patients underwent sentinel lymph biopsy and five underwent axillary clearance. A skin-sparing simple mastectomy was performed in the patient with a phyllodes tumor. Six patients received chemotherapy for breast cancer, and two also received adjuvant radiotherapy (Table 1). The duration of tissue expansion ranged from three to six months, with an average of four months, and an average of 460 $\mathrm{mL}$ of tissue expansion was achieved (range, 390-550 mL) (Fig. 3). The implants ranged in size from 255 to $425 \mathrm{~mL}$ (Fig. 3).

\section{Table 1. Demographic information of the patients who underwent unilateral breast reconstruction, showing pBRA scores}

\begin{tabular}{|c|c|c|c|c|c|c|c|}
\hline Patient & $\begin{array}{l}\text { Age } \\
(y r)\end{array}$ & $\begin{array}{l}\text { Indication for two-stage LD } \\
\text { reconstruction }\end{array}$ & $\begin{array}{c}\text { Breast } \\
\text { procedure }\end{array}$ & $\begin{array}{l}\text { Adjuvant } \\
\text { treatment }\end{array}$ & Cancer staging & $\begin{array}{l}\text { Follow-up } \\
\text { duration (mo) }\end{array}$ & pBRA score ${ }^{a)}$ \\
\hline$A$ & 33 & Patient preference ${ }^{\mathrm{b})}$ & SSM+SLNBX & None & T1NOMO & 20 & 3.8 \\
\hline B & 64 & Caesarean section & $\mathrm{SSM}+\mathrm{AC}$ & $\begin{array}{l}\text { Chemotherapy } \\
\text { Radiotherapy }\end{array}$ & T1CN1M0 & 13 & 2 \\
\hline C & 42 & Caesarean sections & $\mathrm{SSM}+\mathrm{AC}$ & Chemotherapy & T2NOMO & 7 & 5.8 \\
\hline D & 61 & Previous abdominal surgery & SSM+SLNBX & Chemotherapy & T1aN0M0 & 7 & 5.2 \\
\hline E & 45 & Caesarean sections & SSM & None & Phyllodes tumor & 12 & 3.5 \\
\hline $\mathrm{F}$ & 43 & Caesarean sections & $\mathrm{SSM}+\mathrm{AC}$ & Chemotherapy & T2N1M0 & 15 & 7.1 \\
\hline G & 59 & Previous abdominal surgery & $\mathrm{SSM}+\mathrm{AC}$ & Chemotherapy & T2NOMO & 10 & 4.1 \\
\hline $\mathrm{H}$ & 54 & $\begin{array}{l}\text { Previous TRAM flap for opposite } \\
\text { breast reconstruction }\end{array}$ & SSM+SLNBX & None & TisNOMO & 22 & 6.5 \\
\hline । & 28 & Patient preference $e^{b)}$ & $\mathrm{SSM}+\mathrm{AC}$ & $\begin{array}{l}\text { Chemotherapy } \\
\text { Radiotherapy }\end{array}$ & T2N1M0 & 20 & 2.8 \\
\hline
\end{tabular}


Eight patients underwent secondary nipple reconstruction. No major or minor postoperative complications were observed among the nine patients. The average follow-up duration was 13 months (ranging, 7-22 months). No cancer recurrence was reported.

In terms of aesthetic outcome, seven patients scored $<6$ on the pBRA scale, indicating excellent breast symmetry, with the remaining two scoring between 6 and 8, indicating good symmetry (Table 1).

\section{Case A (Fig. 4)}

Case A was a 33-year-old female with no significant prior medi- cal history (Fig. 4A). She was diagnosed with localized right invasive breast cancer. This patient opted for two-stage $\mathrm{LD}$ flap reconstruction instead of transverse rectus abdominis myocutaneous (TRAM) flap reconstruction to avoid abdominal scarring and potential complications in future pregnancies. Skin-sparing mastectomy with a sentinel lymph node biopsy and breast reconstruction were performed (Fig. 4B). During the two-stage reconstruction, a 550-mL Mentor tissue expander (pre-filled with $20 \mathrm{~mL}$ of saline) was placed (Fig. 4C, D). The final staging of the tumor was T1NOM0, and adjuvant chemotherapy was administered. At three months postoperatively, $420 \mathrm{~mL}$ of tissue expansion had been completed (Fig. 4E). A 255-mL Silimed

\section{Fig. 4. Two-stage LD reconstruction in patient A}

(A) Preoperative photograph. (B) The latissimus dorsi (LD) flap skin paddle marking. (C) Subpectoral placement of a 550-mL Mentor expander prefilled with $20 \mathrm{~mL}$ of saline and the LD flap. (D) Tension-free closure of the reconstructed breast. (E) Tissue expansion and size symmetrization stages. (F) One-year postoperative photograph after placement of a $255-\mathrm{mL}$ implant and nipple reconstruction.
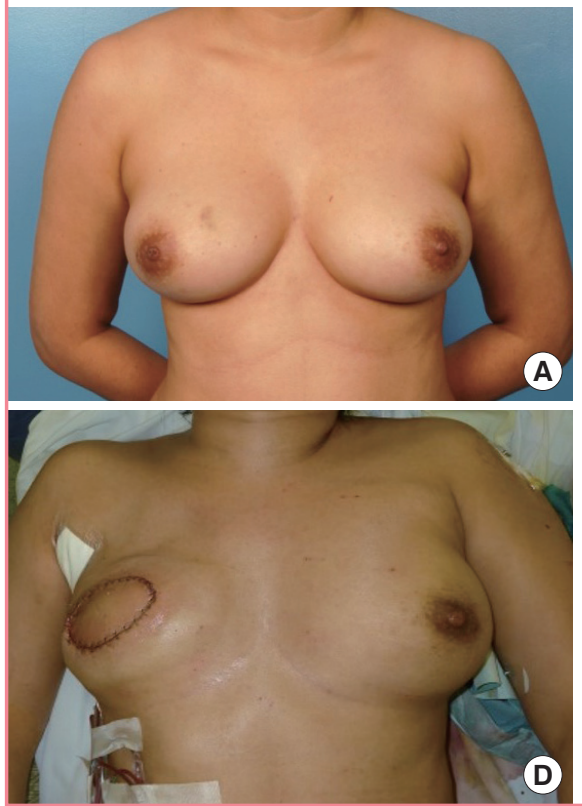
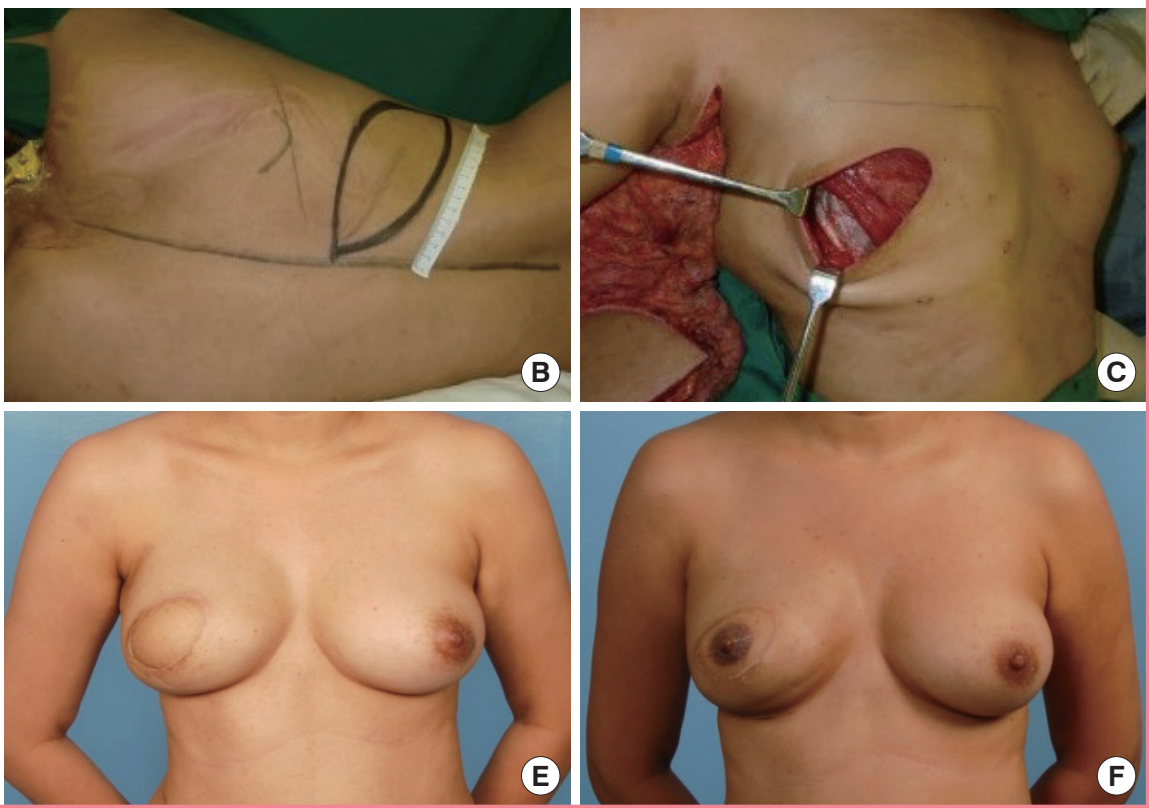

Fig. 5. Two-stage LD reconstruction for patient B

(A) Preoperative photograph. (B) Subpectoral placement of a 550-mL Mentor expander pre-filled with $100 \mathrm{~mL}$ of saline and the latissimus dorsi (LD) flap. (C) Thirteen-month postoperative photograph after placement of a 375-mL implant and nipple reconstruction.
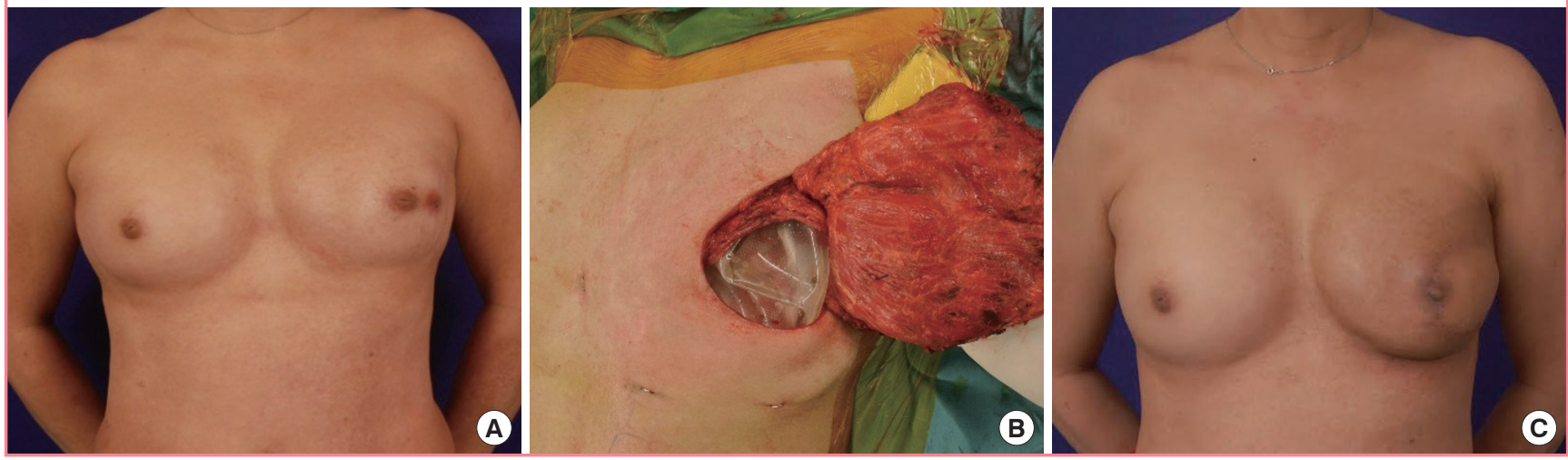
implant was used to replace the expander. Nipple reconstruction and tattooing were performed seven months after the initial operation. At her most recent follow-up visit (20 months postmastectomy), no recurrence was observed, and she was satisfied with the aesthetic results (Fig. 4F).

\section{Case B (Fig. 5)}

Case $\mathrm{B}$ was a 64-year-old female with a history of bilateral breast augmentation with $270-\mathrm{mL}$ round implants and one Caesarean section (Fig. 5A). She was diagnosed with left invasive breast cancer. The patient opted for two-stage LD flap reconstruction instead of a TRAM flap, which might have been complicated by her previous Caesarean section. Skin-sparing mastectomy with axillary clearance and breast reconstruction were performed. During the two-stage reconstruction, a 550-mL Mentor tissue expander (pre-filled with $100 \mathrm{~mL}$ of saline) was placed (Fig. 5B). The final staging of the tumor was T1N1M0, and adjuvant chemotherapy and radiotherapy were administered. A total tissue expansion of $550 \mathrm{~mL}$ had taken place six months postoperatively, after a delay for the radiotherapy. Breast symmetry was achieved after deflating the expander to $380 \mathrm{~mL}$, and a $375-\mathrm{mL}$ Mentor round implant was used to replace the expander. The patient underwent subsequent nipple reconstruction and tattooing. At her most recent follow-up visit (13 months post-mastectomy), no recurrence was noted, and she was satisfied with the aesthetic results (Fig. 5C).

\section{Case C (Fig. 2)}

Case $\mathrm{C}$ was a 42 -year-old female who was diagnosed with invasive right breast cancer (Fig. 2A). Her prior medical history included two Caesarean sections, thus increasing the risk of complications with an abdominal flap. She underwent skin-sparing mastectomy with sentinel lymph node biopsy and two-stage LD reconstruction with an expander. A 550-mL Mentor expander pre-filled with $100 \mathrm{~mL}$ of saline was inserted. The staging revealed the presence of T2NOMO breast cancer, and chemotherapy was administered. Four months postoperatively, $540 \mathrm{~mL}$ of tissue expansion had taken place (Fig. 2B). The tissue expander was deflated to $250 \mathrm{~mL}$ to achieve symmetrization (Fig. 2C). During the implant placement operation, the expander was removed and a $255-\mathrm{mL}$ Mentor implant was placed. Subsequently, the patient underwent nipple reconstruction and tattooing. At one year of follow-up, no cancer recurrence was noted and successful aesthetic results were achieved (Fig. 2D).

\section{DISCUSSION}

The LD flap is a well-established and safe procedure for breast reconstruction [4]. In the Asian population, this flap alone provides adequate volume for reconstructing small to medium breasts. If necessary, more tissue can be obtained using the extended LD flap technique to achieve symmetry $[5,6]$.

Alternatively, a prosthesis can be used, but correctly determining the size is challenging. During a one-stage operation involving an LD flap and an implant, a large skin paddle is used to augment the volume of the mastectomy skin envelope, thereby allowing the largest possible implant to be used for reconstruction. A large implant is necessary because within the first postoperative year, the LD muscle atrophies, and its ultimate contribution to the volume of the breast is unpredictable. However, although symmetry can be achieved with this approach, it is at the expense of additional scars that reduce patient satisfaction. Secondary fat grafting is another option to achieve symmetry in single-stage LD reconstruction [7]. However, this approach has a modest effect on tissue expansion, and the patient has to be motivated and willing to undergo multiple fat grafting sessions. In the presence of an implant, safety is paramount, and we prefer small-volume fat injections using a 16-gauge blunt-tip cannula.

In contrast, the two-stage approach provides time for soft tissue healing and the inevitable atrophy of the LD muscle before a decision is made about the size of the final implant. The first stage of the tissue expansion process, ironically, does not actually aim for expansion, but to rest the skin envelope. This is followed by the second stage, two to three weeks later, in which the skin is gradually expanded to achieve a volume comparable with the opposite side, followed by $20 \%-30 \%$ overexpansion to create a natural degree of ptosis.

This technique removes the need for a large LD skin paddle externally and confines the scars to the nipple-areolar complex, producing a better aesthetic result. Any excess skin is de-epithelialized and used as a soft tissue cover for natural augmentation and to improve breast projection. Furthermore, it reduces the need for extended LD flaps, which are commonly associated with increased donor site morbidity.

Other options for two-stage breast reconstruction include using a tissue expander with acellular dermal matrix. In the first stage, the tissue expander is placed subpectorally, and its exposed lower pole is covered with acellular dermal matrix. The steps of tissue expansion and implant exchange are similar to our technique, but without an LD flap. This approach may achieve symmetry, provided the skin envelope is well preserved and vascularized [8]. In our practice, skin-sparing mastectomy is performed by a separate oncologic team. The skin envelope is at times excessively thinned and ischemic. By using a LD flap, postoperative complications, such as implant extrusion, seroma formation, and infection, are reduced [9]. Furthermore, the skin paddle of 
the LD flap provides ample skin for the nipple-areolar-complex defect, ensuring that the future nipple is level with the opposite side.

If a pre-existing implant is present in the contralateral breast, the shape of the implant should match that of the existing one. In patients $B$ and one other patient with previous bilateral round breast implants, a similar implant was used to match the contralateral breast. An anatomical implant would not have had the upper pole fullness of a round implant.

In patients undergoing radiotherapy, the purpose of the staging procedure is twofold: first, it counteracts the contracture caused by radiation by keeping the skin stretched, and secondly, skin quality is improved by recruiting neighboring chest skin, and the skin is gradually softened with fat grafting in the later stages [10]. In such cases, the tissue expansion proceeds at a slower pace in order to overcome skin tightness [11].

Admittedly, the two-stage reconstruction is a more time-consuming process with multiple clinical visits and an additional operation, and it may therefore seem less preferable to the patient. However, in our experience, when the long-term benefits of this technique are explained, patients accept the additional inconvenience in order to achieve the desirable results.

\section{REFERENCES}

1. Giacalone PL, Bricout N, Dantas MJ, et al. Achieving symmetry in unilateral breast reconstruction: 17 years experience with 683 patients. Aesthetic Plast Surg 2002;26:299302.

2. Slavin SA. Improving the latissimus dorsi myocutaneous flap with tissue expansion. Plast Reconstr Surg 1994;93:811-24.

3. Vrieling C, Collette L, Bartelink E, et al. Validation of the me- thods of cosmetic assessment after breast-conserving therapy in the EORTC "boost versus no boost" trial. EORTC Radiotherapy and Breast Cancer Cooperative Groups. European Organization for Research and Treatment of Cancer. Int J Radiat Oncol Biol Phys 1999;45:667-76.

4. Hammond DC. Latissimus dorsi flap breast reconstruction. Clin Plast Surg 2007;34:75-82.

5. Germann G, Steinau HU. Breast reconstruction with the extended latissimus dorsi flap. Plast Reconstr Surg 1996;97: 519-26.

6. Lee JW, Chang TW. Extended latissimus dorsi musculocutaneous flap for breast reconstruction: experience in Oriental patients. Br J Plast Surg 1999;52:365-72.

7. Bonomi R, Betal D, Rapisarda IF, et al. Role of lipomodelling in improving aesthetic outcomes in patients undergoing immediate and delayed reconstructive breast surgery. Eur J Surg Oncol 2013;39:1039-45.

8. Kim JY, Connor CM. Focus on technique: two-stage implantbased breast reconstruction. Plast Reconstr Surg 2012;130 (5 Suppl 2):104S-115S.

9. Luce EA, Adams RL, Chandler RG, et al. Tissue expander versus tissue expander and latissimus flap in morbidly obese breast reconstruction patients. Plast Reconstr Surg Glob Open 2015;3:e323.

10. Serra-Renom JM, Munoz-Olmo JL, Serra-Mestre JM. Fat grafting in postmastectomy breast reconstruction with expanders and prostheses in patients who have received radiotherapy: formation of new subcutaneous tissue. Plast Reconstr Surg 2010;125:12-8.

11. Alani H, Shantour S. Breast reconstruction by tissue expander after radiotherapy: when the skin does not expand, the rib cage is at risk. Eur J Plast Surg 2014;37:333-8. 